This is the accepted version of the following article:

Fernández-Gavela A., Herranz S., Chocarro B., Falke F., Schreuder E., Leeuwis H. Heideman R.G., Lechuga L.M.. Full integration of photonic nanoimmunosensors in portable platforms for on-line monitoring of ocean pollutants. Sensors and Actuators, B: Chemical, (2019). 297. 126758: - . 10.1016/j.snb.2019.126758,

which has been published in final form at https://dx.doi.org/10.1016/j.snb.2019.126758 @ https://dx.doi.org/10.1016/j.snb.2019.126758. This manuscript version is made available under the CC-BY-NC-ND 4.0 license http://creativecommons.org/licenses/by-nc-nd/4.0/ 


\section{Accepted Manuscript}

Title: Full integration of photonic nanoimmunosensors in portable platforms for on-line monitoring of ocean pollutants

Authors: Adrián Fernández-Gavela, Sonia Herranz, Blanca Chocarro, Floris Falke, Erik Schreuder, Henk Leeuwis, René G. Heideman, Laura M. Lechuga

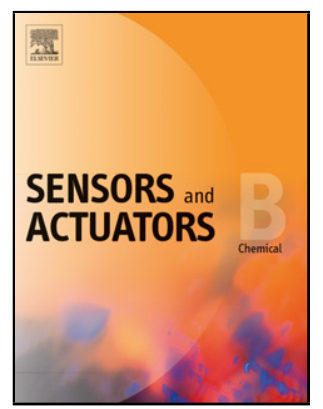

PII: S0925-4005(19)30681-1

DOI: https://doi.org/10.1016/j.snb.2019.126758

Reference: SNB 126758

To appear in: $\quad$ Sensors and Actuators B

Received date: $\quad 30$ October 2018

Revised date: $\quad 2$ April 2019

Accepted date: $\quad 30$ April 2019

Please cite this article as: Fernández-Gavela A, Herranz S, Chocarro B, Falke F, Schreuder E, Leeuwis H, Heideman RG, Lechuga LM, Full integration of photonic nanoimmunosensors in portable platforms for on-line monitoring of ocean pollutants, Sensors and amp; Actuators: B. Chemical (2019), https://doi.org/10.1016/j.snb.2019.126758

This is a PDF file of an unedited manuscript that has been accepted for publication. As a service to our customers we are providing this early version of the manuscript. The manuscript will undergo copyediting, typesetting, and review of the resulting proof before it is published in its final form. Please note that during the production process errors may be discovered which could affect the content, and all legal disclaimers that apply to the journal pertain. 


\title{
Full integration of photonic nanoimmunosensors in portable platforms for on-line monitoring of ocean pollutants
}

\author{
Adrián Fernández-Gavela ${ }^{a}$, Sonia Herranz ${ }^{a}$, Blanca Chocarro ${ }^{a}$, Floris Falke ${ }^{b}$, Erik Schreuder ${ }^{b}$, Henk \\ Leeuwis $^{\mathrm{b}}$, René G. Heideman ${ }^{\mathrm{b}}$, Laura M. Lechuga*a \\ ${ }^{a}$ Nanobiosensors and Bioanalytical Applications Group, Catalan Institute of Nanoscience and Nanotechnology (ICN2), CSIC, BIST \\ and CIBER-BBN. Campus UAB, Bellaterra, 08193 Barcelona, Spain \\ ${ }^{\mathrm{b}}$ Lionix International. PO Box 456, 7500 Al Enschede, The Netherlands \\ * Corresponding author. Email: laura.lechuga@icn2.cat
}

Research highlights

- Novel microfluidic cartridge for an optical sensor has been designed and fabricated

- Rotary valve has been implemented for an autonomous fluid delivery system

- Detection of Irgarol 1051 and Tetracycline, as proof of concept, accomplishing EU legislation

- Optical and data acquisition integration in aMZI sensor for on-line monitoring

\begin{abstract}
We have developed a photonic nano-immunosensor platform for the on-site analysis of harmful organic ocean pollutants, intended to be allocated in stand-alone buoys. The main aim is bringing the monitoring tools directly to the contaminated place, resulting in cost and time savings as compared to the standard analytical techniques. As sensor we have employed an integrated asymmetric Mach-Zehnder interferometer (aMZI) of micro/nano dimensions, based on silicon photonic technology. In order to obtain a multiplexed system, a four-channel microfluidic cell has been designed, manufactured and incorporated in the miniaturized sensor. Additionally, a microfluidic delivery module enabling automatic sample analysis has been designed, evaluated and assembled. Moreover, we have implemented the optical interconnections of the sensor chip by fiber optics, as well the electronics and the required software and data processing. Pollutant detection is based on a competitive immunoassay using bioreceptors previously biofunctionalized on the aMZI sensor arms and incubation with a specific antibody. As proof of concept, two types of pollutants have been analysed: the biocide Irgarol 1051, and the antibiotic Tetracycline. Results show limits of detection in the range of few $\mathrm{ng} / \mathrm{mL}$, accomplished the European legislation.
\end{abstract}

Keywords: Optical biosensor; lab-on-a-chip; integrated optics; microfluidics; ocean pollution.

\section{Introduction}

The exponential degradation of the marine environment is an alarming concern at worldwide level. Anthropogenic activities have been causing a profound negative impact in our environment during last decades due to the growing human and industrial activities. Thousands of chemical and biological compounds are thrown to the marine environment annually. These pollutants enter the marine environment via atmospheric deposition, as a result of runoff, direct discharges of wastes and wastewaters from land into the sea, through accidents or illegal discharges from ships and companies. The early detection and control of the pollution is essential to manage environmental degradation and protecting the quality of the oceans for the world future. Recent advances in biosensor devices have demonstrated the possibility to develop portable and reliable analytical devices for real-time and on-site monitoring of 
different types of contaminants [1-3]. In contrast to chemical analysis, which identifies compounds on the basis of their physical properties, biosensors are based on biomolecular interactions between analytes and their specific bioreceptors. Biosensors transduce such biomolecular recognition into an electrical signal that can be further processed. Their advantages for environmental analysis are mainly related to their high sensitivity and selectivity $[4,5]$ while operating with real and untreated samples.

Moreover, biosensors can be integrated in portable platforms opening the door for a truly on-site environmental monitoring.

Within the European Union BRAAVOO project (Biosensors, Reporters and Algal Autonomous Vessels for Ocean Operation) the main objective was to develop innovative solutions for real-time and in-situ evaluation of marine pollutants of high impact and difficult to detect (http://www.braavoo.org). One of the solutions offered by the project was the development of a dedicated and highly sensitive nanophotonic immunosensor device, combined with the required microfluidics, optical and electronic modules, to be embedded in a marine data buoy for continuous ocean monitoring.

The specific focus in photonic nano-immunosensors is justified because photonic biosensors present undisputed advantages with respect to other detection schemes, such as electrochemical or piezoelectric. For example, they are not easily disturbed by electromagnetic interferences, can potentially be used in aggressive environments, provide excellent miniaturization capabilities via optoelectronic integration, and offer possibilities for multiplexing and multi-sample analysis in an unamplified and label-free scheme $[6,7]$ which is important for the purpose of on-site analysis in a buoy. More specifically, sensors based on integrated optics are exceptionally amenable to chip integration, multiplexing and miniaturization [8-10]. The most employed mechanism for these photonic biosensors is the evanescent wave sensing, where the binding of analyte to their specific bioreceptors, immobilized to the waveguide sensor surface, causes a refractive index change near the surface within the evanescent field of the light travelling in the optical waveguide sensor. These analytical devices not only enable high sensitive but also label-free detection of analytes in real time. Several competitive evanescent wave biosensors have been developed so far, such as MachZehnder interferometers (MZI) [11-13], microring resonators [14] or bimodal waveguides [15], among others [7].

In this work, we have employed a multiplexed asymmetric Mach-Zehnder interferometer (aMZI) sensor [16], which is easily integrated in lab-on-a-chip platforms, by enabling combination of both fluidic handling and optical analysis onto a single chip.

Because we project to offer a multiplexed system for the simultaneous analysis of several pollutants present in the same sample, we have also developed a novel polymer fluidic cell matching the aMZI multiplex sensor chip. In order to be able to deploy the nano-immunosensor/microfluidics cartridge in the buoy for on-line monitoring we needed to design a dedicated electronic module. Furthermore, a novel rotary valve system has been implemented to achieve an appropriate fluidic connection within the available space in the on-site modules, while having low energy consumption, and avoiding a complex standard fluidic delivery system. Figure 1 illustrates all the components needed for the complete integration of the nanoimmunosensor platform in the buoy. 
In addition of the instrumental development and as a proof of concept of the feasibility of the a-MZI biosensor technology for pollution monitoring, we have focused in the detection of two types of pollutants, the antibiotic Tetracycline and the antifouling paint booster biocide Irgarol 1051, due to their impact in the marine pollution.

Antibiotics are used extensively in human and veterinary medicine, and also in aquaculture, in order to prevent or treat microbial infections. Antibiotic residues from different sources (households, pharmaceutical industries, and hospitals) enter into municipal sewage but unfortunately, they are only partially eliminated at sewage treatment plants and, as consequence, many residues are released into the environment. The uncontrolled exposure to antibiotic residues increases the appearance of antibiotic-resistant bacteria, which represents one of the most serious and growing human health threat worldwide. Moreover, since they are bioactive substances, acting highly effectively at low doses, their presence can cause ecological harm in non-target terrestrial and marine aquatic life. We have focused our attention in one of the most important group of antibiotics used in aquaculture as are tetracyclines.

Irgarol 1051 is a protective algaecide (biocide) used in antifouling paints to prevent attachment and growth of biofouling organisms in the aquatic environment. As consequence of its intensive use, traces of Irgarol 1051 have been widely detected in the sea. Irgarol 1051 belongs to the s-triazine group of compounds and due to their broad toxicity, is potentially toxic toward valuable aquatic species, causing significant environmental problems. The European Water Framework Directive (WFD) 2013/39/EC included Irgarol 1051 as a priority pollutant in the field of water policy, setting its maximum allowable concentration at only few $\mathrm{ng} / \mathrm{L}$.

As both pollutants to be detected are of low-molecular weight (444.45 Da for Tetracycline and 253.37 Da for Irgarol 1051, respectively), a competitive assay format has been employed for their label-free and real-time evaluation. In this type of immunoassay, we immobilise the analyte or a similar protein derivative on the sensor surface (bioreceptor layer). The water sample to be tested (which contains the pollutants) is incubated during few minutes with a fixed concentration of the corresponding specific antibody. Once incubated, the sample was passed over the biosensor surface and an interaction of the remaining free antibodies of the incubated solution with the bioreceptor layer takes place. The signal obtained is inversely proportional to the concentration of the pollutant in the sample. Figure 2 shows the scheme of a competitive immunoassay and the expected output data. As we have to re-use the bioreceptor layer during as many cycles as possible for on-line actuation, a regeneration step of the sensor biosurface is also designed by dissociating the receptor-antibody interaction with appropriate regeneration solutions.

\section{Material and methods}

\subsection{Materials}

Ethanol, acetone, hydrochloric acid (37\%) and nitric acid (65\%) were purchased from Panreac (Spain). Carboxyethysilanetriol sodium salt (CTES) was purchased from ABCR (Germany). N-(3-Dimethylaminopropyl)-N'-ethylcarbodiimide hydrochloride (EDC), NHydroxysulfosuccinimide sodium salt (sulfo-NHS), ethanolamine, components for 
phosphate buffer (PB) and phosphate buffer saline (potassium phosphate monobasic, sodium phosphate dibasic, sodium chloride and potassium chloride), sodium acetate (SA), 2-(N-Morpholino)ethanesulfonic acid (MES) and sodium hydroxide ( $\mathrm{NaOH}$ ) were purchased from Sigma-Aldrich (Germany). The receptors DoxAc10-AD (conjugate of a tetracycline derivative with dextran) and cona-4e (conjugate of an Irgarol 1051 derivate with conalbumin protein), as well as the antiserum specific for tetracycline (As256, rabbit polyclonal anti-tetracycline serum) and antiserum specific for Irgarol 1051 (As87, rabbit polyclonal anti-Irgarol 1051 serum) were produced by Nanobiotechnology for Diagnostics Nb4D group, at IQAC-CSIC (Spain). Irgarol 1051 was kindly provided by the same group. Tetracycline hydrochloride was purchased from Abcam (USA). Deionized water from a Milli-DI ${ }^{\circledR}$ Water Purification System (Merk Millipore, USA) was always employed.

Vertical Cavity Surface Emitting Laser (VCSEL) (U-L-M Photonics) was purchased from Philips Technologie GMBH (The Netherlands). Flow cell was manufactured by Microtec GMBH (Germany). Fluidic connection, 4-way edge connectors (Multiflux Linear Connector 4-way + Edge connector) was acquired from Dolomite Microfluidics (United Kingdom). 12-channel fiber array was purchased from Xio Photonics B.V. (The Netherlands). Stepper motor (42BYGHW811 NEMA-17 Bipolar 48mm Stepper) included in Rotary Valve was acquired from 3D Printer Components (The Netherlands). Syringe pumps (NE-501) was purchased from New Era Pump Systems Inc (USA). Peristaltic pumps (P625) was acquired from Instech Laboratories, Inc (USA). OSROM system was implemented by Lionix International (The Netherlands).

\section{2. $a \mathrm{MZI}$ sensor device}

The aMZI photonic sensor was manufactured at a clean room foundry using a dedicated Triplex ${ }^{\mathrm{TM}}$ technology from Lionix International [17]. The core of the MZI waveguides is fabricated with stoichiometric silicon nitride $\left(\mathrm{Si}_{3} \mathrm{~N}_{4}\right) 103 \mathrm{~nm}$ thick and 1 $\mu \mathrm{m}$ width, with $\mathrm{SiO}_{2}$ as claddings. At the sensor arm, the $\mathrm{SiO}_{2}$ cladding was removed, opening a sensing window of $500 \mu \mathrm{m} \times 675 \mu \mathrm{m}$, such that the evanescent field of the light travelling in the aMZI can interact with the environment, which in our case is a sample fluid. Within the MZI sensor, a Y-splitter was used to split the propagating light into two separate arms with a path length of roughly $6.25 \mathrm{~mm}$. One arm functions as reference and the other arm as sensor. Both waveguides are recombined again with another Y-splitter and lead to the output of the chip. Small induced delays to the phase of the light in the sensor arm result in a measurable power fluctuation at the output of the chip. These fluctuations at the output are a result of constructive or destructive interference between the light going through the sensor arm and the reference arm. These phase delays are a result of changes in the effective refractive index in the sensor arm caused by the biointeractions taking place at the sensor surface. As Figure 3 shows, the asymmetry of the MZI sensors was obtained by adding a few microns of additional path length to the reference waveguide. Adding an asymmetry has the benefit that it enables an output interference pattern, which becomes wavelength dependent, making it much easier to track the direction of the occurring refractive index change [16].

A Vertical Cavity Surface Emitting Laser (VCSEL) light source $(\lambda=850 \mathrm{~nm}$ ) was used to incoupling the light in the sensors and also to sweep the asymmetric Mach-Zehnder over a bandwidth of $3 \mathrm{~nm}$, resulting in an output interference pattern that monitors 
not only the intensity change for a specific wavelength but also the direction in which the phase shift occurs. Optical characterization of the aMZI device shows that very high sensitivities, up to a minimum change of $10^{-7}$ RIU (Refractive Index Units), can be obtained [16].

\subsection{Competitive Immunoassays for pollutant detection}

Target compound (tetracycline or Irgarol 1051) recognition is based on a competitive immunoassay format in which the target analyte and the receptor compete for a fixed amount of antibody ( $\mathrm{Ab}$ ) binding sites as explained above. Thus, an antiserum specific for tetracycline or an antiserum specific for Irgarol 1051 was added to the sample to be analysed (solution concentration of the target ranges from 0 to $100 \mu \mathrm{g} / \mathrm{L}$ ) and, after initiate a continuous flow of phosphate buffer saline (PBS, pH 7.4) over the sensor, 150 $\mu \mathrm{L}$ of the mixture (sample+Ab) was injected into the flow cell. Specificity and selectivity of the employed polyclonal antibodies to detect Irgarol1051 were previously tested by ELISA immunoassays [18]. The free Ab interacts with the immobilized receptor, producing a sensor signal, inversely proportional to the target concentration in the sample. Finally, the surface was regenerate by injecting a $\mathrm{NaOH}$ solution for 2 min $(25$ $\mu \mathrm{L} / \mathrm{min}, 10 \mathrm{mM}$ and $50 \mathrm{mM}$ for tetracycline and Irgarol 1051, respectively), leaving the biosurface ready for the evaluation of a new sample.

\section{Results and discussion}

\subsection{Integration of the aMZI platform}

The full integration of the aMZI sensors in a portable platform includes: a) the flow cell and the flow delivery system b) optical connections $\mathrm{c}$ ) electronics and data processing. In order to have a multiassay sensor, a single chip with six aMZI sensors has been manufactured in which three sensors were used for multiplexed measures. The additional three sensors in the chip are added for flexibility of the sensor platform. They can be used to measure a reference sample or one of the samples in threefold, for example. To transfer the samples to each sensor individually, a specific flow cell was designed. The width of an aMZI sensor is $500 \mu \mathrm{m}$ and they are placed on a pitch of $700 \mu \mathrm{m}$. This leaves $200 \mu \mathrm{m}$ of space for a separating structure including tolerances on alignment. This translates in high restrictions on the minimal dimensions and accuracy of the manufacturing process for the flow cell. It must be also taken into account that an easy exchange of the sensor cartridge is mandatory for an on-line system. For that, the flow cell was designed as two independent pieces, which allow an easy and fast assembly. As Figure 4 shows, the bottom piece was considered taking into account the chip dimensions $(10 \times 10 \mathrm{~mm})$ and the optical connection needed to guarantee a robust device. Also, in order to get an efficient connection between the flow delivery system and the flow cell, the top piece of the cartridge was planned considering two commercial 4-way edge connector, which allows an easy connection. Additionally, individual microchannels were implemented for getting a multiplexed sensor as Figure 5 shows. This microfluidic cartridge design, provides a robust system needed for the on-line monitoring using a stand-alone buoy in the ocean. For the flow cell fabrication, a suitable manufacturing process was implemented by usage of Rapid Micro Product Development (RMPD technology), which structure 
channels in (soft)polyacrylate material with micron accuracy. This accuracy in combination with a high tolerance alignment rods enabled an alignment between a "top" flow cell and the sensor chip, positioned in a "bottom" cartridge, within 50 microns, as Figure 4 shows. The RMPD process uses a hard polyacrylate for structuring of the top flow cell and bottom chip cartridge. Furthermore, a $200 \mu \mathrm{m}$ thin soft acrylate layer was structured on the top flow cell and functions as reversible sealant and flow channel, when the flow cell was compressed against the sensor chip, as Figure 5 shows.

The width of the sealant between the sensors was $100 \mu \mathrm{m}$. In compression, four individual flow paths of $100 \mu \mathrm{m}$ in height were created, three of them flow over the three sensors separately (internal volume $0.2 \mu \mathrm{L}$ ) and one flows over three additional sensors (internal volume $1.0 \mu \mathrm{L}$ ), as Figure 6 and Figure 7 show, respectively.

The optical connection of the sensor cartridge was facilitated by alignment and gluing of a 12-channel fiber array to the edge of the chip, by using a UV-curing adhesive (single mode, polarisation dependent input fiber), as Figure 8 shows. The manufactured flow cell has an integrated clamp to fixate the fibers and prevent tension on the fibers from pulling at the glued interface between the fiber array and the sensor chip. This optical connection provides a robust in/out-coupling method, which could even allow operating over turbulences in this ocean condition.

\subsection{Biofunctionalization protocol}

Once the integration of the aMZI sensors in a robust cartridge was achieved, this one was employed for demonstrating its biosensor performance. Previously to that, the inclusion of the bioreceptors at the sensor surfaces was done before the sensors were placed in the microfluidic cartridge.

We have employed a biofunctionalization protocol based on wet chemistry silanization and subsequent covalent receptor immobilization [19]. For silanization process, the sensors were first thoroughly cleaned by consecutively rinsing with acetone, ethanol and deionized water. Then, they were sonicated for $10 \mathrm{~min}$ in ethanol: $\mathrm{HCl}(1: 1, \mathrm{v} / \mathrm{v})$, rinsed with deionized water and dried under nitrogen stream.

Cleaned aMZI sensors were oxidized for $5 \mathrm{~min}(99 \%, 40 \mathrm{kHz}$ and $100 \mathrm{~W}$, Femto oxygen plasma, Electronic Diener), immersed in a $15 \%$ nitric acid solution at $75{ }^{\circ} \mathrm{C}$ for $25 \mathrm{~min}$, rinsed generously with deionized water and immediately immersed in an aqueous $0.5 \%$ solution of the silane (CTES) for $1 \mathrm{~h}$ in order to obtain a silaned monolayer at the surface. Finally, the CTES-treated chips were rinse with deionized water, dried under nitrogen stream and thermally cured at $110^{\circ} \mathrm{C}$ for $1 \mathrm{~h}$.

At this point, aMZI sensors were aligned with and fixed to the fiber array. Then, the aMZI sensors were positioned in the microfluidic cartridge and the immobilization of the receptor (DoxAc10-AD for tetracycline sensor or cona-4e for Irgarol 1051, respectively) was carried out using an in-flow immobilization approach: after initiate continuous flow $(20 \mu \mathrm{L} / \mathrm{min})$ of deionized water, the carboxylic groups on the sensor surface (CTES moieties) were activated with $200 \mathrm{mM} \mathrm{N}$-(3-Dimethylaminopropyl)-N'ethylcarbodiimide hydrochloride (EDC) and $50 \mathrm{mM} \mathrm{N}$-Hydroxysulfosuccinimide sodium salt (sulfo-NHS) solution in $100 \mathrm{mM}$ MES buffer containing $0.5 \mathrm{M} \mathrm{NaCl}$, at pH 5.0 (150 $\mu \mathrm{L}, 20 \mu \mathrm{L} / \mathrm{min})$, followed by incubation with the receptor solution $(20 \mu \mathrm{g} / \mathrm{mL}, 150 \mu \mathrm{L}$ at $10 \mu \mathrm{L} / \mathrm{min})$. Finally, an ethanolamine solution (1 M, pH 8.5) was flowed onto the sensor surface ( $25 \mu \mathrm{L} / \mathrm{min}, 120 \mathrm{~s}$ ) to deactivate residual carboxylic groups and to remove 
electrostatically bound ligands. Figure 9 shows a scheme of the functionalization protocol.

\subsection{Irgarol and Tetracycline aMZI biosensors}

Four different buffers were evaluated as the most appropriate solvent for the receptor solution: i) phosphate buffer (PB, pH 6), ii) PBS, pH 7.4, iii) sodium acetate ( $\mathrm{SA}, 10 \mathrm{mM}$, $\mathrm{pH} 4.5-5)$ and iv) MES buffer $(10 \mathrm{mM}, \mathrm{pH} 4.5-6)$. Suitable coupling conditions were determined by injecting the receptor (DoxAc10-AD or cona-4e, $10 \mu \mathrm{g} / \mathrm{mL}$ ) over a sensor surface that has not been activated with EDC/sulfo-NHS, and measuring the response due to electrostatically bound ligand. If the receptor is pre-concentrated onto the surface, an increase immobilization yield is expected.

The highest electrostatic pre-concentration of DoxAc10-AD and cona-4e were observed for MES buffer at pH 5 and PBS pH 7.4 respectively (data not shown). Thus, both MES and SA buffers ( $10 \mathrm{mM}, \mathrm{pH} 5)$ were evaluated for the covalent attachment. Figure 10 shows, as example, the functionalization of an aMZI sensor with DoxAc10-AD receptor. As can be observed, 79\% of the amount of the bound receptor $(\Delta(I)=1080 \pm$ 30 u.a.) is immobilized $(\Delta(\mathrm{II})=850 \pm 20$ u.a.). Non-covalent immobilized receptor was washed out by flowing ethanolamine $(1 \mathrm{M}, \mathrm{pH} 8.5)$ over the surface.

Specific receptor-antibody interaction for DoxAc10-AD/As256 and cona-4e/As87 pairs was then evaluated. As expected, an increase of the sensor response was observed by increasing the concentration of the antibody present in the solution. A 1:3000 dilution factor of antiserum for both As256 and As87 was selected for the competitive immunoassays. With this sera concentration is possible to achieve an appropriate signal intensity (in terms of signal/noise ratio) in absence of target compound ( $\triangle$ (As87) $=355 \pm 20$ u.a. and $\Delta(A s 256)=193 \pm 9$ u.a.). Finally, the response of the sensors for solutions of increasing concentrations of the target compound (tetracycline or Irgarol 1051) was evaluated. Figure 11 shows the competitive calibration curve obtained for both interferometric immunosensors (calibration curve obtained using a 4-parameter logistic model).

Tetracycline immunosensor achieved a Limit of Detection, LOD (concentration of target compound for which the signal was inhibited by $10 \%$ of the sensor signal range) of only $0.04 \mu \mathrm{g} / \mathrm{L}$ and an IC50 value of $0.19 \mu \mathrm{g} / \mathrm{L}$, with a dynamic range (analyte concentrations that produce a sensor signal between 20 and $80 \%$ of sensor signal range) from 0.08 to $0.5 \mu \mathrm{g} / \mathrm{L}$. Irgarol 1051 immunosensor shown a LOD of only 0.07 $\mu \mathrm{g} / \mathrm{L}$ and an IC50 value of $1.6 \mu \mathrm{g} / \mathrm{L}$, with a dynamic range from 0.2 to $12 \mu \mathrm{g} / \mathrm{L}$. Finally, in order to use the sensor in an unattended platform in the ocean, for a long time, a regeneration cycles were evaluated as section 2.3 described. Additionally, highly concentrated Ab solutions present long-term stability (over 4 weeks) at $4-8{ }^{\circ} \mathrm{C}$. As example, Figure 12 shows a blank sample measures in Irgarol 1051 sensor, followed by a regeneration cycle, where the baseline was recovered in three sensors, us expected. The sensor reproducibility after regeneration for both sensors has been evaluated, reaching the same sensors response up to ten cycles. The good performance of the results indicates that both aMZI photonic biosensors can be suitable for an on-line monitoring of both analytes.

\subsection{Steps toward lab-on-chip sensor allocation in a buoy}


A key objective in the design of a marine monitoring buoy is to achieve a platform that is large enough to host the solar power and battery packs and at the same time having a system that can be packaged for easy transport. Also, the buoy must contain enough space inside to accommodate the biosensors and all its components to allow autonomous operation. In adition, in order to avoid degradation of the Ab probe, the final portable biosensing module should include a temperature control system, which allows storing $\mathrm{Ab}$ probe at $4-8^{\circ} \mathrm{C}$. Additionally, a data acquisition and telemetry system are needed for communication to and from the buoy, store data and other information transmitted by the biosensors.

In order to make portable our biosensing module, for allocation in the buoy, the fluidics for sample distribution and manipulation had to be downscaled and made autonomous. For that, once the biosensor detection of Irgarol 1051 and tetracycline has been demonstrated at laboratory level, as well as in real sea samples without the need of any pre-treatment [20], a portable microfluidics module was designed and developed offering as best solution a novel Rotary Valve (RV).

The RV consists of a single microfluidic glass chip having a predefined network of internal fluid channels and a passive mixing unit. The channels were interconnected depending on the position by a Teflon selector rotor positioned above the chip. Additionally, the RV has the ability to change multiple fluidic paths simultaneously allowing multiplexing. Its cuts heavily down on required components and dead volumes while maintaining a reasonable system size.

The network of fluidic channels and the passive mixer unit of the RV are etched using $\mathrm{HF}$ acid in the glass microchip. The entrance of each fluidic channel was connected to the outside through powder blasted holes. These holes were pressed against an O-ring seal when the microchip was placed into a holder. This holder act as adapter and establishes the link to standard fluidic connectors. The fluidic channels inside the glass chip all lead to the center of the chip and their outputs are positioned in a circular configuration, matching the layout of the rotor. These outputs are on the opposite side of the chip compared to the entrances as Figure 13 shows. These connections were in contact with a polished Teflon rotor, which was pressed against the glass microchip to achieve a leak tight connection. In this Teflon rotor, channels were micromachined, which depending on the position of the rotor (or rotation angle) provides a connection between at least two different fluidic channel outputs, as Figure 14 shows. The glass microchip can be crafted such that it has complex fluidic pathways and passive functionalities can be added. The custom rotor enables multiplexing of the amount of connections made between the different channels.

The RV designed supports the full immunoassay requirements including the following steps: 1) Dilution of sample (sea water) with DI water; 2 ) Mixing of the diluted sample with one of the different antibodies (100:1 ratio); 3) Filling an injection loop (150 $\mu \mathrm{L})$ with the sample/DI/antibody mixture; 4) Preparation/ flushing of sensor surface with buffer; 5) Injection of sample loop to one of the three sensors using buffer; 6) Dilution of the regeneration fluid with DI water to a ratio compatible with the surface chemistry of the sensor; 7) Filling an injection loop $(150 \mu \mathrm{L})$ with the regeneration solution/DI water mixture; 8) Flushing of the internal lines with or without the sample loop using buffer or DI water.

As Figure 15 shows, positioning of the rotor is facilitated by a stepper motor. A lever connected to the Teflon rotor together with an optical switch determines the "zero" or 
home position. Fluid transfer to and from the RV was done by three syringe pumps for controlled injection of $\mu \mathrm{L}$ of antibody and two peristaltic pumps for general fluid transport in the $\mu \mathrm{L} / \mathrm{min}$ range. All were placed onto an aluminium baseplate on suspension together with holders for fluid containers, the sensor flow cell and a mini PC. Pumps and RV were controlled by home-made LabVIEW software, which can work in manual or automated mode.

As operation RV proof of concept in automated mode, a script was implemented for a complete cycle of measurements, from the mixture of the sample to the regeneration cycle. For that, a generic serum was employed by using PBS as buffer solution and $\mathrm{NaOH} 50 \mathrm{nM}$ as regeneration solution. As Figure 16 shows, a whole cycle has been completed in automated mode, observing in all cases the RV dispensed the fluid required. These results demonstrated the total autonomous of the fluid delivery system, approaching the full integration of the photonic sensors in portable platforms for on-line monitoring.

To complete the system, an OSROM system houses all electronics for the management of optics and sensor data acquisition: VCSEL current driver; VCSEL temperature control; eight photodiodes with amplifier and DAQ data acquisition card. The entire system was placed inside a pelicase for easy, robust transport and for minimize the ocean perturbations, due the variation of current or waves.

\section{Conclusions}

A full integration of aMZI immunosensor cartridges has been accomplished for the online monitoring of sea water pollutants. A new design of aMZI biosensor chips have been done taking into account the need to ensemble a flow cell for multiplexing capabilities. A polymer flow cell with four independent microchannels has been implemented in a cartridge by using RMPD technology. The full integration of the aMZI chip inside the cartridge has been tested and applied to a competitive immunoassay for Tetracycline and Irgarol 1051 detection, obtaining a LOD of $0.04 \mu \mathrm{g} / \mathrm{L}$ and 0,07 $\mu \mathrm{g} / \mathrm{L}$, respectively, which together with an appropriate dynamic range, ensure the applicability for pollutant detection accomplishing the limits set by the EU legislation. In order to achieve a full integration of the photonic immunosensors for unattended operation in a buoy, an OSROM system with all the electronics for the management of optics and sensor data acquisition and processing was used. Furthermore, to avoid a complex microfluidic system, with large required volumes, lots of dead volumes, required space and energy consumption, a novel RV has been developed and integrated in a flow delivery system. We performed a preliminary test of the whole optical and electrical system on board of the buoy in a marine by checking that such modules were correctly operating, which confirms us the suitability and robustness of our approach.

As future steps, we will need to perform extended set of experiments including all the modules and the operation with real samples delivered directly by the sampling system of the buoy and validation of the results with the corresponding analytical techniques.

\section{Acknowledgements}


This work has been funded by the 7FP (EU, BRAAVOO Grant Agreement No 614010. The authors would like to thank MicroTEC company (Duisburg, Germany) for the technical support and for the fabrication of the microfluidic cartridge. We acknowledge Nanobiotechnology for Diagnostics Nb4D group at IQAC-CSIC (Barcelona, Spain) for the productions of the Irgarol 1051 immunoreagents.

ICN2 acknowledges support of the Spanish MINECO through the Severo Ochoa Centers of Excellence Program under Grant SEV-2013-0295. The ICN2 is funded by the CERCA programme/ Generalitat de Catalunya.

\section{References}

[1] P. D'Orazio, Biosensors in clinical chemistry - 2011 update, Clin. Chim. Acta. 412 (2011) 1749-1761. doi:10.1016/j.cca.2011.06.025.

[2] W. Zhang, A. Mohamed, D. Liu, D. Du, Y. Lin, Trends in Analytical Chemistry Nanomaterial-based biosensors for environmental and biological monitoring of organophosphorus pesticides and nerve agents, Trends Anal. Chem. 54 (2014) 1-10. doi:10.1016/j.trac.2013.10.007.

[3] B. Chocarro-Ruiz, A. Fernández-Gavela, S. Herranz, L.M. Lechuga, Nanophotonic label-free biosensors for environmental monitoring, Curr. Opin. Biotechnol. 45 (2017) 175-183. doi:10.1016/j.copbio.2017.03.016.

[4] B. Van Dorst, J. Mehta, K. Bekaert, E. Rouah-Martin, W. De Coen, P. Dubruel, R. Blust, J. Robbens, Recent advances in recognition elements of food and environmental biosensors: A review, Biosens. Bioelectron. 26 (2010) 1178-1194. doi:10.1016/j.bios.2010.07.033.

[5] T. Rinken, ed., State of the Art in Biosensors - Environmental and Medical Applications, InTech, 2013. doi:10.5772/56353.

[6] J. Homola, Surface plasmon resonance sensors for detection of chemical and biological species, Chem. Rev. 108 (2008) 462-93. doi:10.1021/cr068107d.

[7] M.C. Estevez, M. Alvarez, L.M. Lechuga, Integrated optical devices for lab-on-achip biosensing applications, Laser Photon. Rev. 6 (2012) 463-487. doi:10.1002/lpor.201100025.

[8] P. Kozma, F. Kehl, E. Ehrentreich-Förster, C. Stamm, F.F. Bier, Integrated planar optical waveguide interferometer biosensors: A comparative review, Biosens. Bioelectron. 58 (2014) 287-307. doi:10.1016/j.bios.2014.02.049.

[9] A.F. Gavela, D.G. García, J.C. Ramirez, L.M. Lechuga, Last advances in siliconbased optical biosensors, Sensors (Switzerland). 16 (2016). doi:10.3390/s16030285.

[10] E. Kim, M.D. Baaske, F. Vollmer, Towards next-generation label-free biosensors: recent advances in whispering gallery mode sensors, Lab Chip. 17 (2017) 11901205. doi:10.1039/C6LC01595F. 
[11] P. V. Lambeck, Remote opto-chemical sensing with extreme sensitivity: Design, fabrication and performance of a pigtailed integrated optical phase-modulated Mach-Zehnder interferometer system, Sensors Actuators, B Chem. 61 (1999) 100-127. doi:10.1016/S0925-4005(99)00283-X.

[12] B. Sepúlveda, J.S. del Río, M. Moreno, F.J. Blanco, K. Mayora, C. Domínguez, L.M. Lechuga, Optical biosensor microsystems based on the integration of highly sensitive Mach-Zehnder interferometer devices, J. Opt. A Pure Appl. Opt. 8 (2006) S561-S566. doi:10.1088/1464-4258/8/7/S41.

[13] T. Chalyan, R. Guider, L. Pasquardini, M. Zanetti, F. Falke, E. Schreuder, R.G. Heideman, C. Pederzolli, L. Pavesi, Asymmetric Mach-Zehnder interferometer based biosensors for Aflatoxin M1 detection, Biosensors. 6 (2016). doi:10.3390/bios6010001.

[14] M. Iqbal, M.A. Gleeson, B. Spaugh, F. Tybor, W.G. Gunn, M. Hochberg, T. BaehrJones, R.C. Bailey, L.C. Gunn, Label-free biosensor arrays based on silicon ring resonators and high-speed optical scanning instrumentation, IEEE J. Sel. Top. Quantum Electron. 16 (2010) 654-661. doi:10.1109/JSTQE.2009.2032510.

[15] K.E. Zinoviev, A.B. González-Guerrero, C. Domínguez, L.M. Lechuga, Integrated bimodal waveguide interferometric biosensor for label-free analysis, J. Light. Technol. 29 (2011) 1926-1930. doi:10.1109/JLT.2011.2150734.

[16] T. Chalyan, L. Pasquardini, F. Falke, M. Zanetti, R. Guider, D. Gandolfi, E. Schreuder, C. Pederzolli, R.G. Heideman, L. Pavesi, Biosensors based on $\mathrm{Si}_{3} \mathrm{~N}_{4}$ asymmetric Mach-Zehnder interferometers, 9899 (2016) $98991 S$. doi:10.1117/12.2227695.

[17] R. Heideman, M. Hoekman, E. Schreuder, TriPleX-based integrated optical ring resonators for lab-on-a-chip and environmental detection, IEEE J. Sel. Top. Quantum Electron. 18 (2012) 1583-1596. doi:10.1109/JSTQE.2012.2188382.

[18] B. Ballesteros, D. Barceló, F. Sanchez-Baeza, F. Camps, M.P. Marco, Influence of the hapten design on the development of a competitive ELISA for the determination of the antifouling agent Irgarol 1051 at trace levels, Anal. Chem. (1998). doi:10.1021/ac980241d.

[19] A.B. González-Guerrero, M. Alvarez, A. García, C. Domínguez, L.M. Lechuga, Journal of Colloid and Interface Science A comparative study of in-flow and micro-patterning biofunctionalization protocols for nanophotonic silicon-based biosensors, (2012). doi:10.1016/j.jcis.2012.10.040.

[20] B. Chocarro-Ruiz, S. Herranz, A. Fernández-Gavela, J. Sanchís, M. Farré, M.P. Marco, L.M. Lechuga, Interferometric nanoimmunosensor for label-free and real-time monitoring of Irgarol 1051 in seawater, Biosens. Bioelectron. 117 (2018) 47-52. doi:10.1016/j.bios.2018.05.044. 


\section{Figure captions:}

- Figure 1. Scheme of the design for the nano-immunosensor full system integration in a buoy for untended operation.

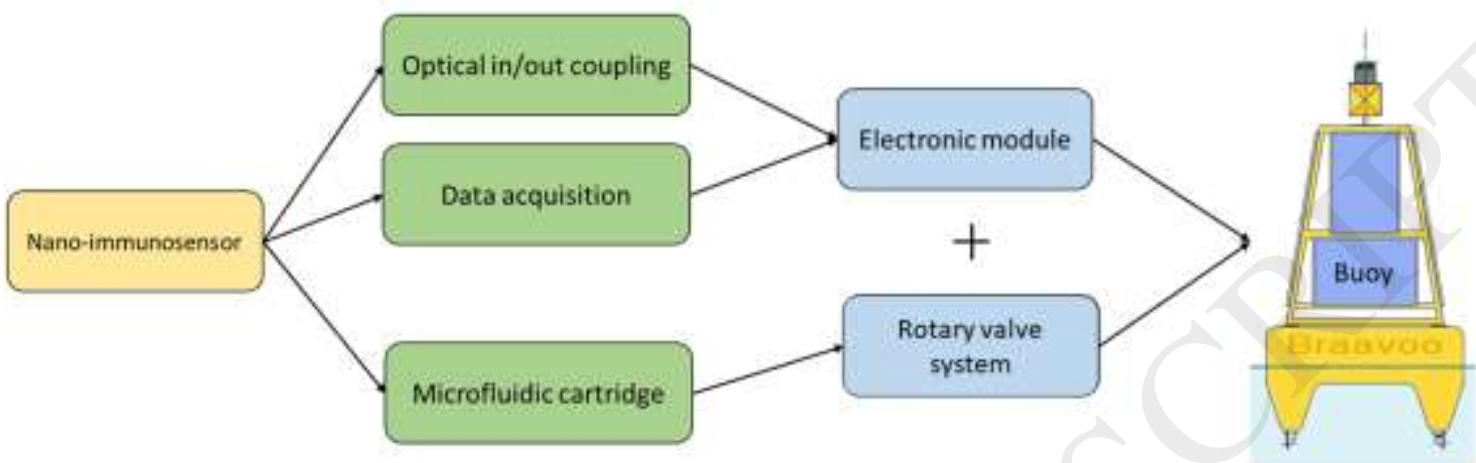

- Figure 2. A: Scheme of a competitive immunoassay. B: Evaluation of the signal and calibration curve provided by a competitive immunoassay.

A

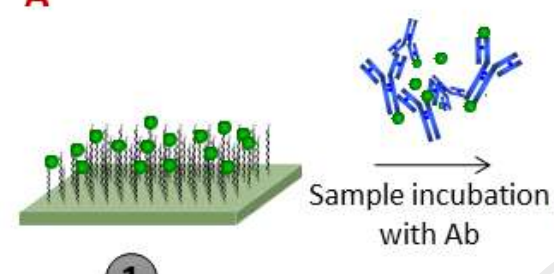

(1)

Receptors biofunctionalization

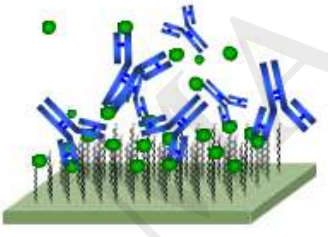

(2)

Biointeraction of free $\mathrm{Ab}$ with receptors

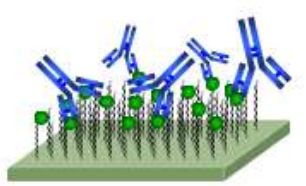

(3)

Biosensing result

B

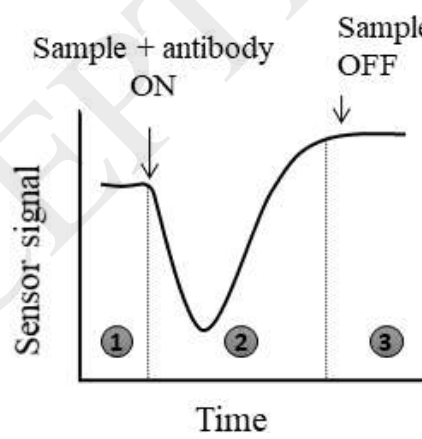

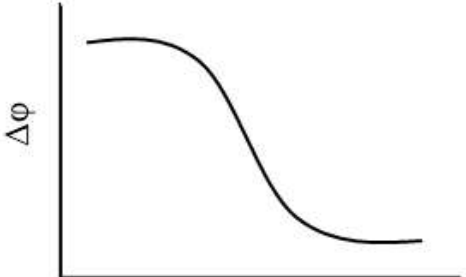

$\log$ [analyte]

- Figure 3. Left: Picture shows the design of the aMZI sensors employed. In red the waveguides, blue indicates the areas where the $\mathrm{SiO} 2$ cladding is removed. The additional path length can be observed at the right of the scheme. Right: Photo of the aMZI chip. 

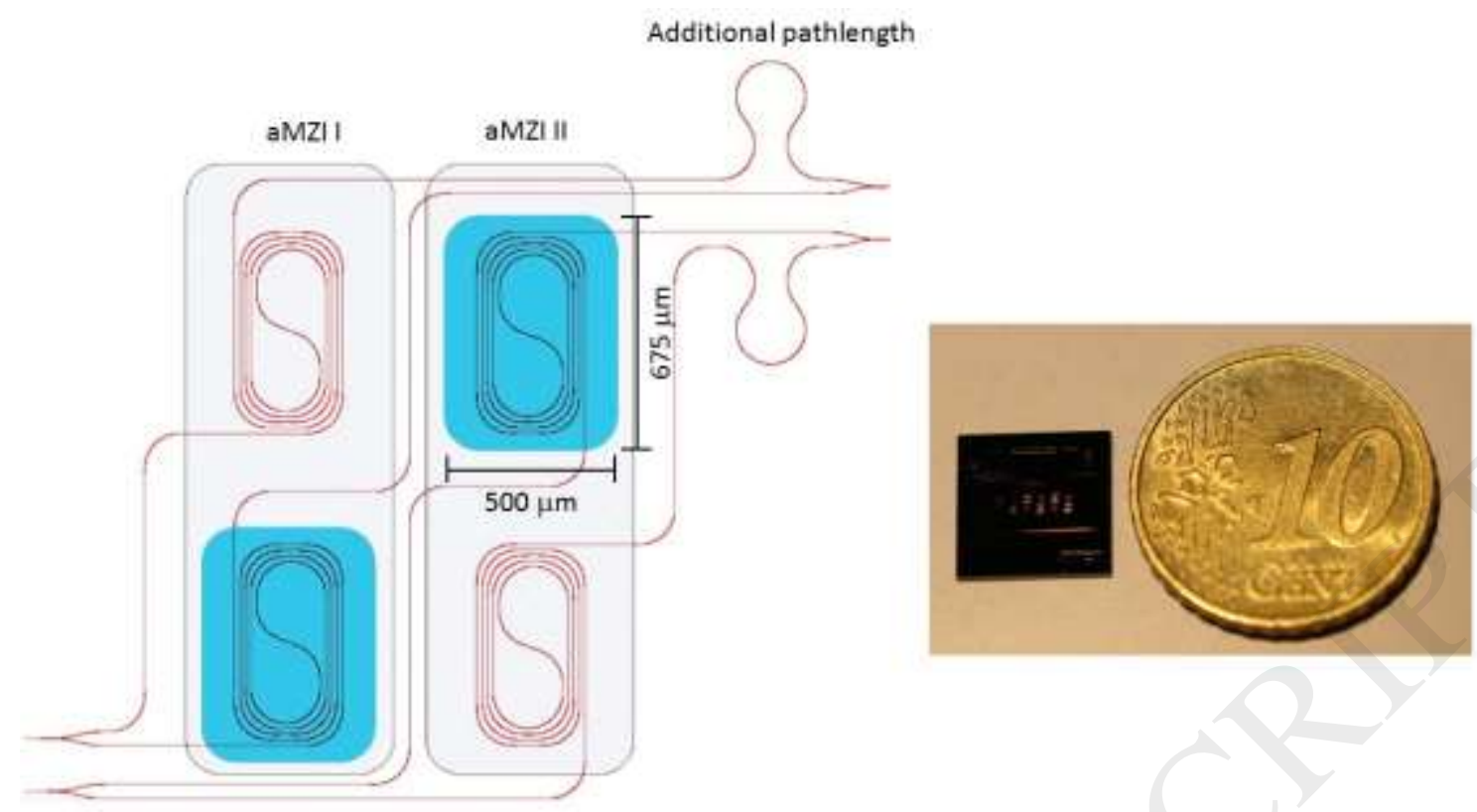

- Figure 4. Left: 3D render of the flow cell assembly. 2 edge connectors are mounted against the edge of the top flow cell which is hovering above the bottom chip cartridge. The chip cartridge is loaded with a chip (grey) connected to a fiber optic array (blue). Right: Photo of the aMZI chip assembly into the cartridge.
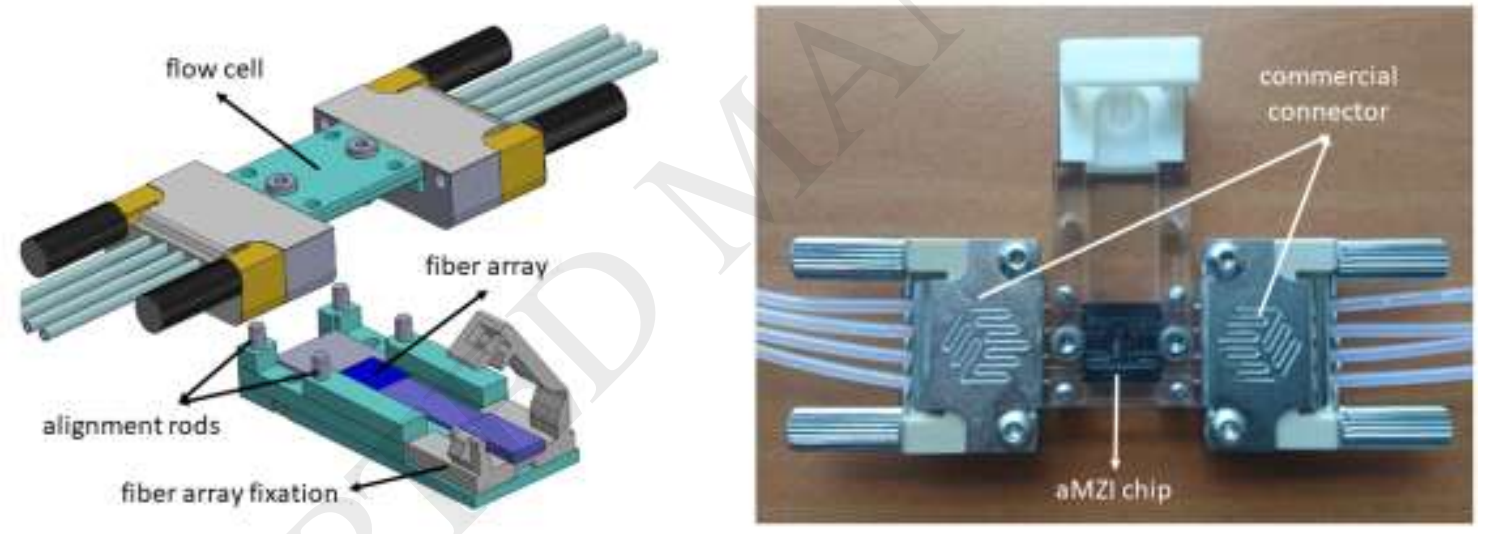

- Figure 5. Left: Transparent 2D view of the top flow cell showing the internal fluidic pathways leading from the edge of the flow cell to the center area and location of the sensor chip. In order to avoid an overpressure in the fluid outlet, which was reflected in the measured signals, an output channels thicker than input were designed. Right: 3D render of the same top cell, in grey is observed the soft acrylate printed seal layer. 

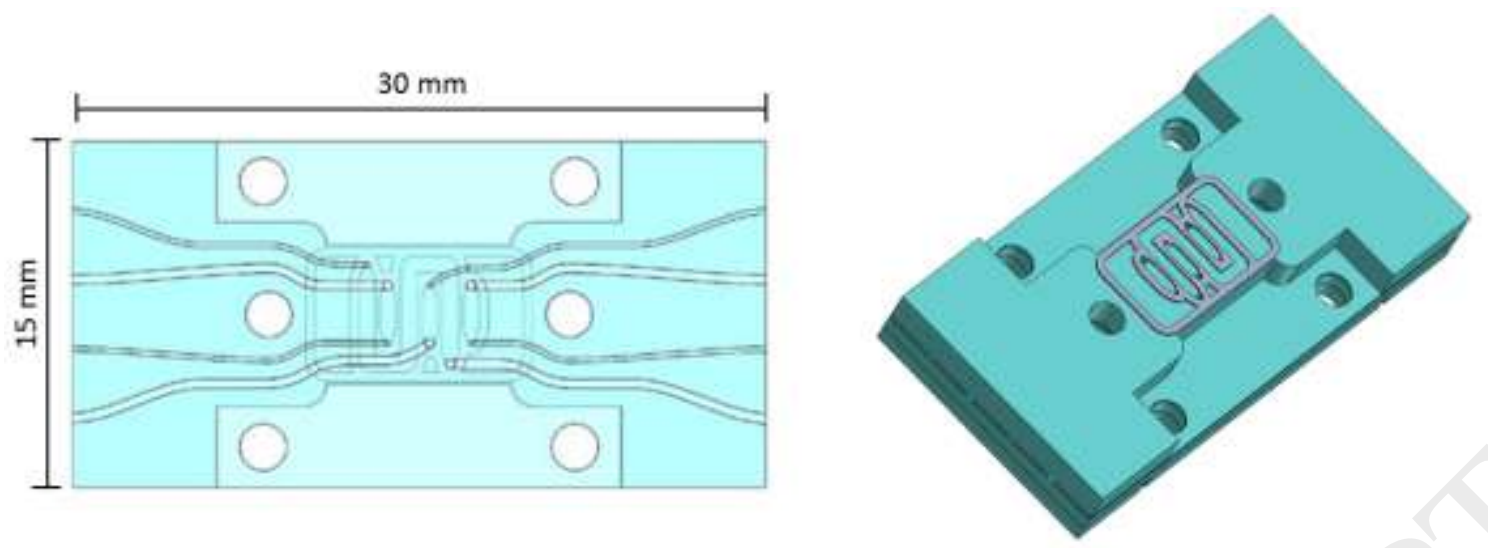

- Figure 6. Cross section of the flow cell. Left: 8-internal channels (4 as inlet and 4 as outlet) leading from the edge connectors to the individual sensors. In orange, the compressed sealant between the sensors (red) on the chip (grey). The black lines are the alignment rods. Right: A close up of the soft acrylate sealing layer pressed against the chip surface forming a barrier between the sensors and simultaneously the flow channels over the sensors. The circles are the fluidic entrance and exit points on the edges and sensor locations (where the cladding $\mathrm{SiO}_{2}$ layer is removed) aligned within the flow channels.
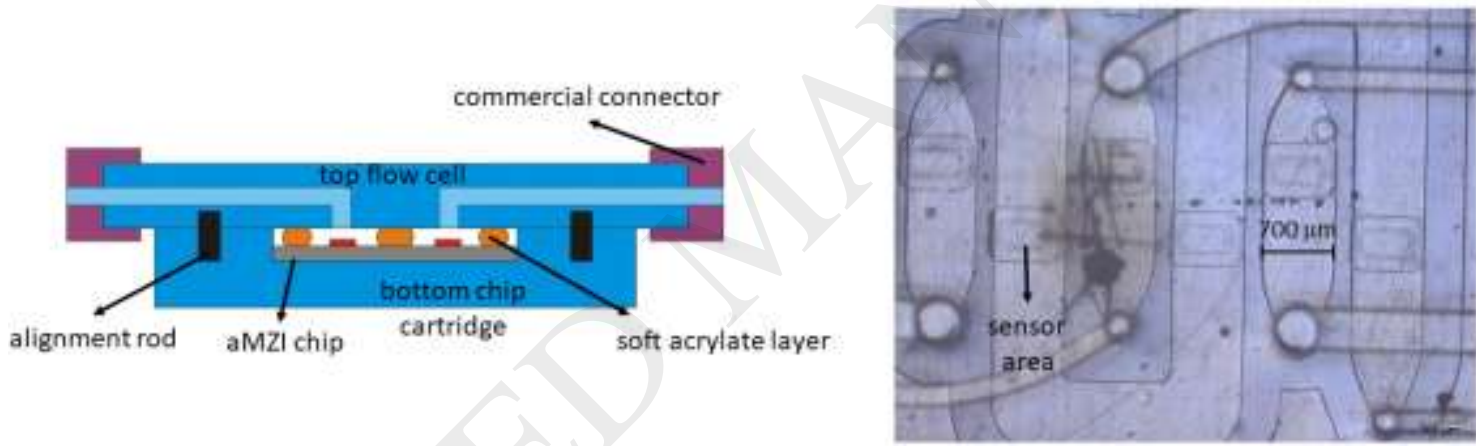

- Figure 7. Overview of the sensor chip layout and flow paths over the different sensors. The three Mach-Zehnders sensors are encircled. The active area of each sensor, indicated by the turquoise colour, was positioned in an individual flow path and ensures that each sensor can be used to measure a different contaminant. Three more sensors are included in this design. They were positioned in the larger surrounding flow path and can be used to simultaneously measure a single contaminant sample in threefold. 


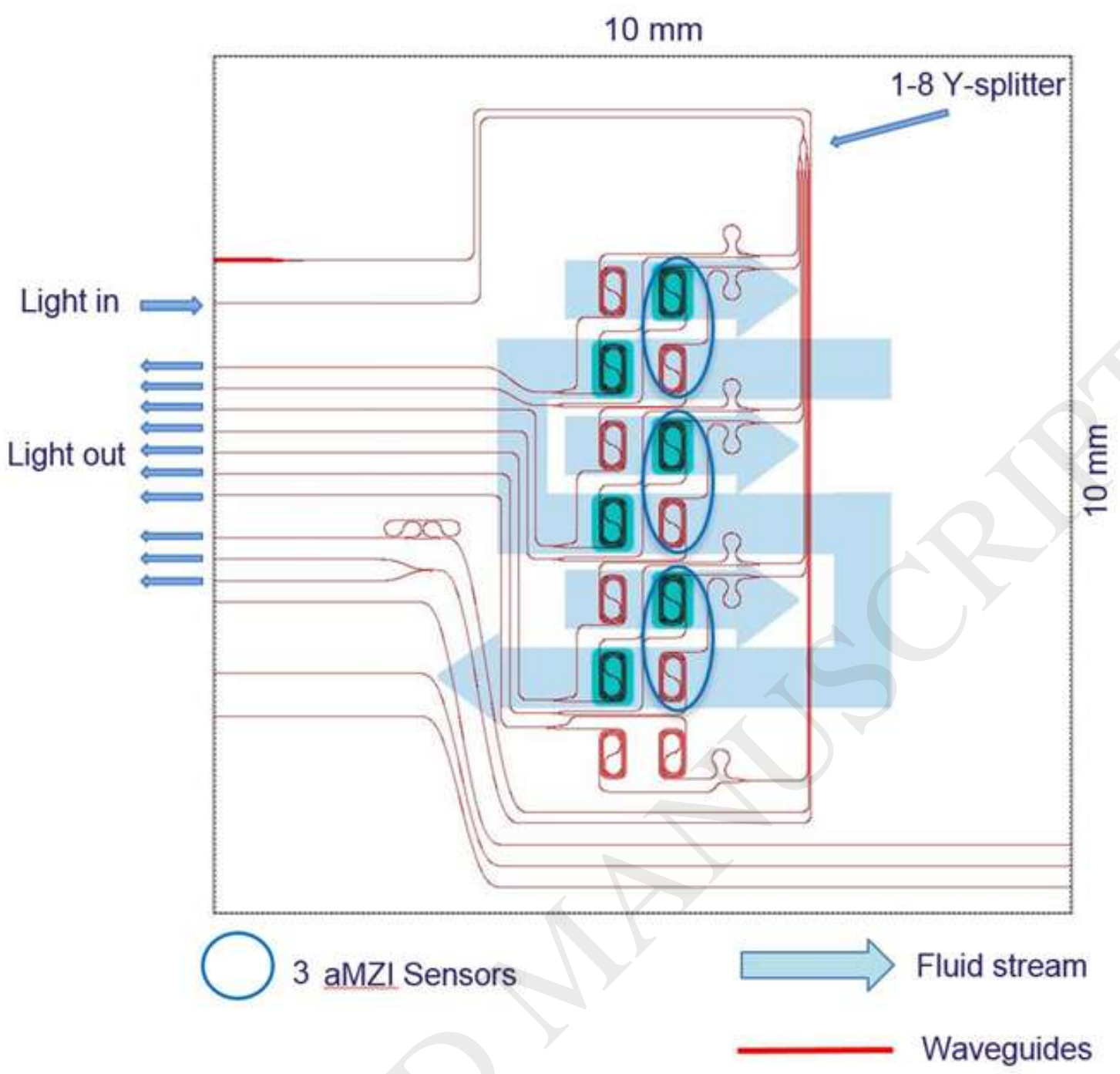

- Figure 8. Photography of an aMZI-chip fixed to a fiber array by using a UV-curing adhesive. 


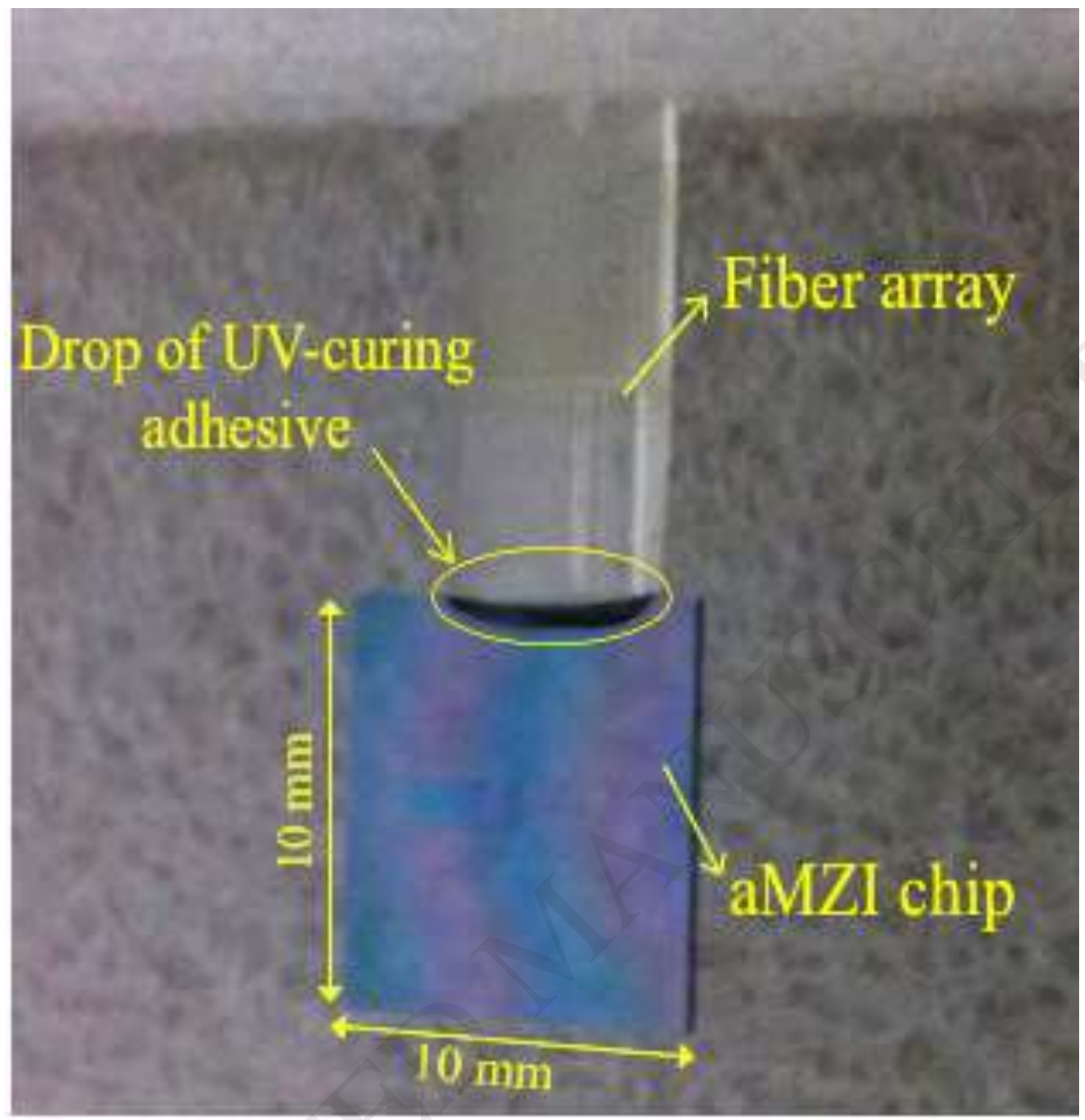

- Figure 9. Protocol for the aMZI sensor surface biofunctionalization.

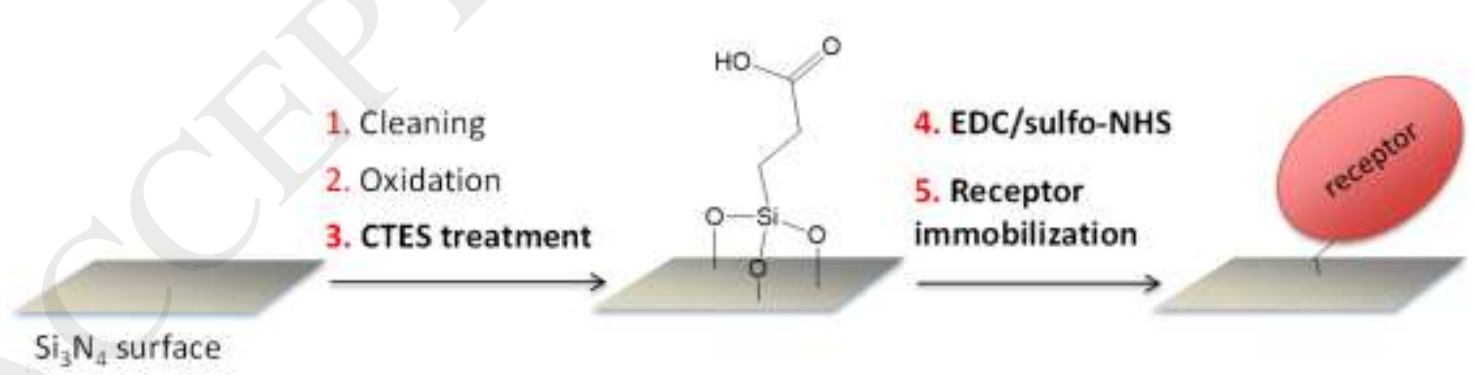

- Figure 10. In-flow immobilization of the receptor DoxAc10-AD (related to tetracycline) onto a CTES-modified aMZI chip. $\Delta$ (I): amount of receptor preconcentrated onto the surface; $\Delta$ (II): amount of receptor covalently immobilized onto the surface. 


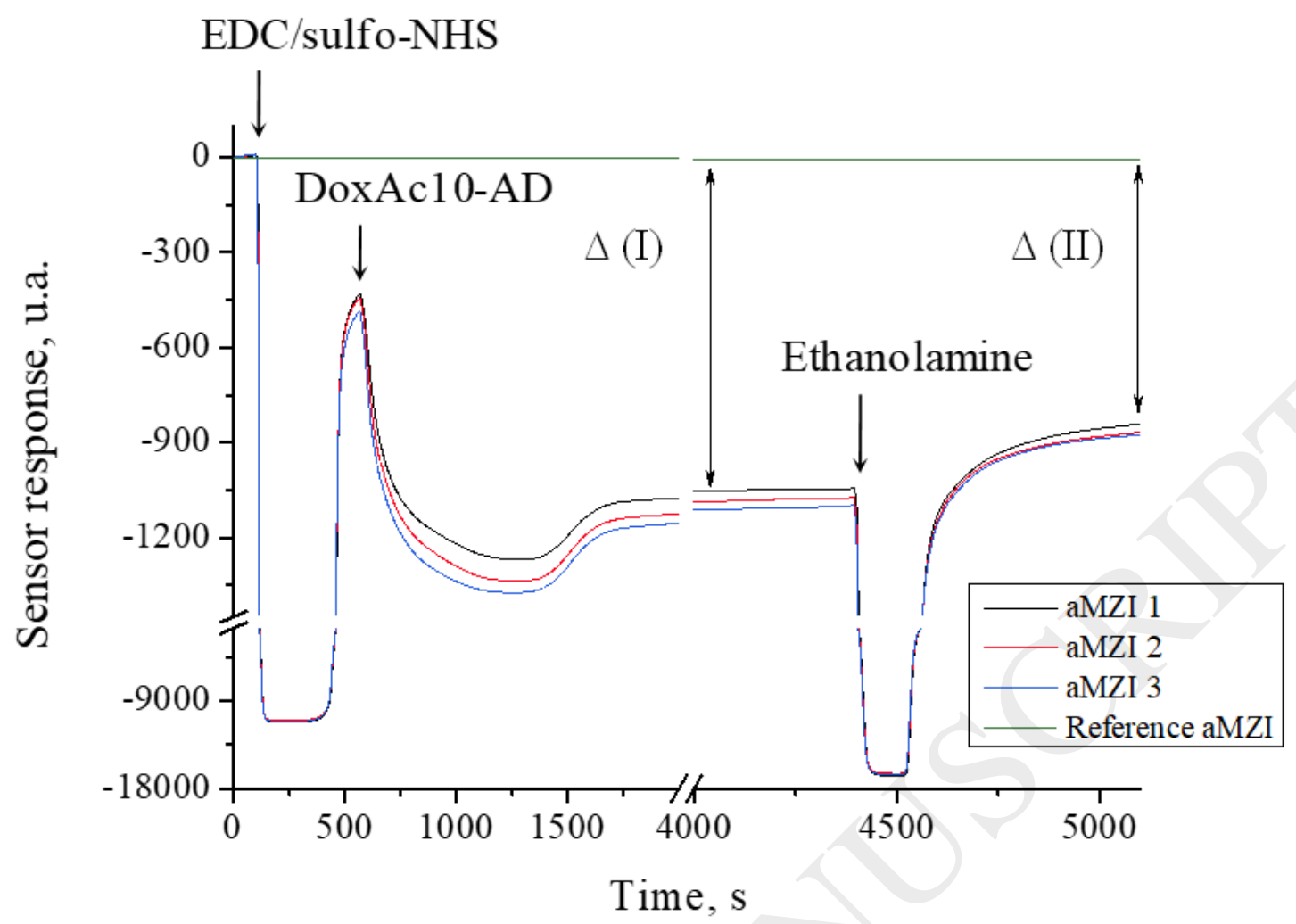

- Figure 11. Calibration curve (4-parameter logistic fitting). Top: tetracycline (standard solutions prepared in PBS). Bottom: Irgarol 1051 aMZI immunosensors (standard solutions prepared in $20 \%$ seawater).
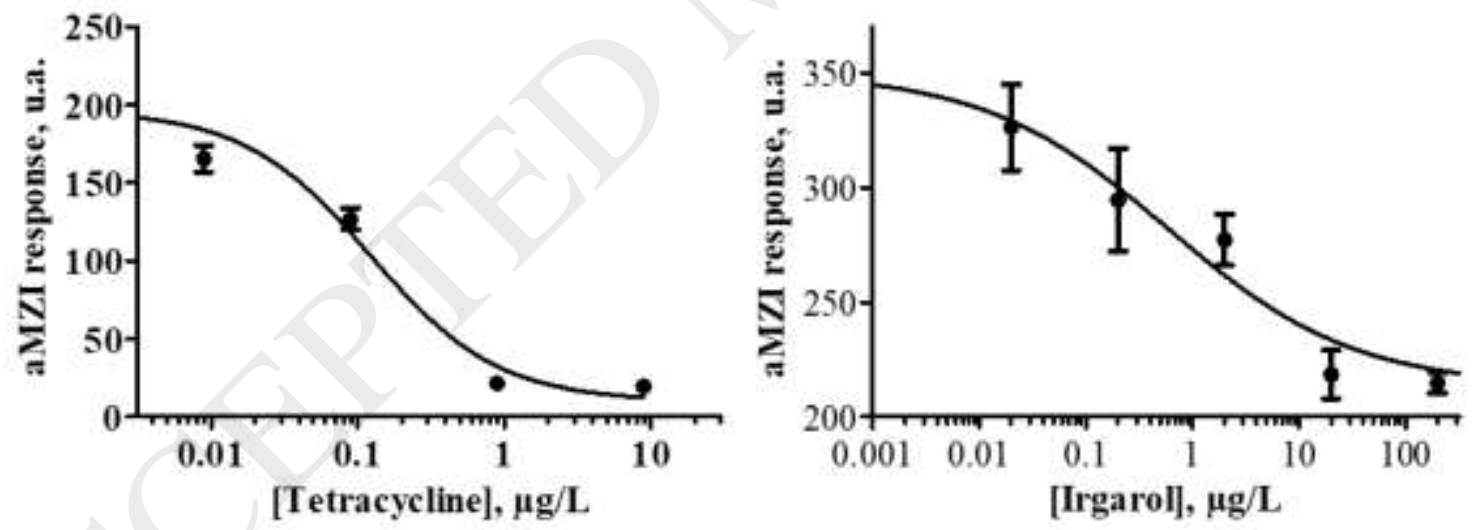

- Figure 12. Regeneration cycle for the Irgarol 1051 sensor after injecting a blank sample. 


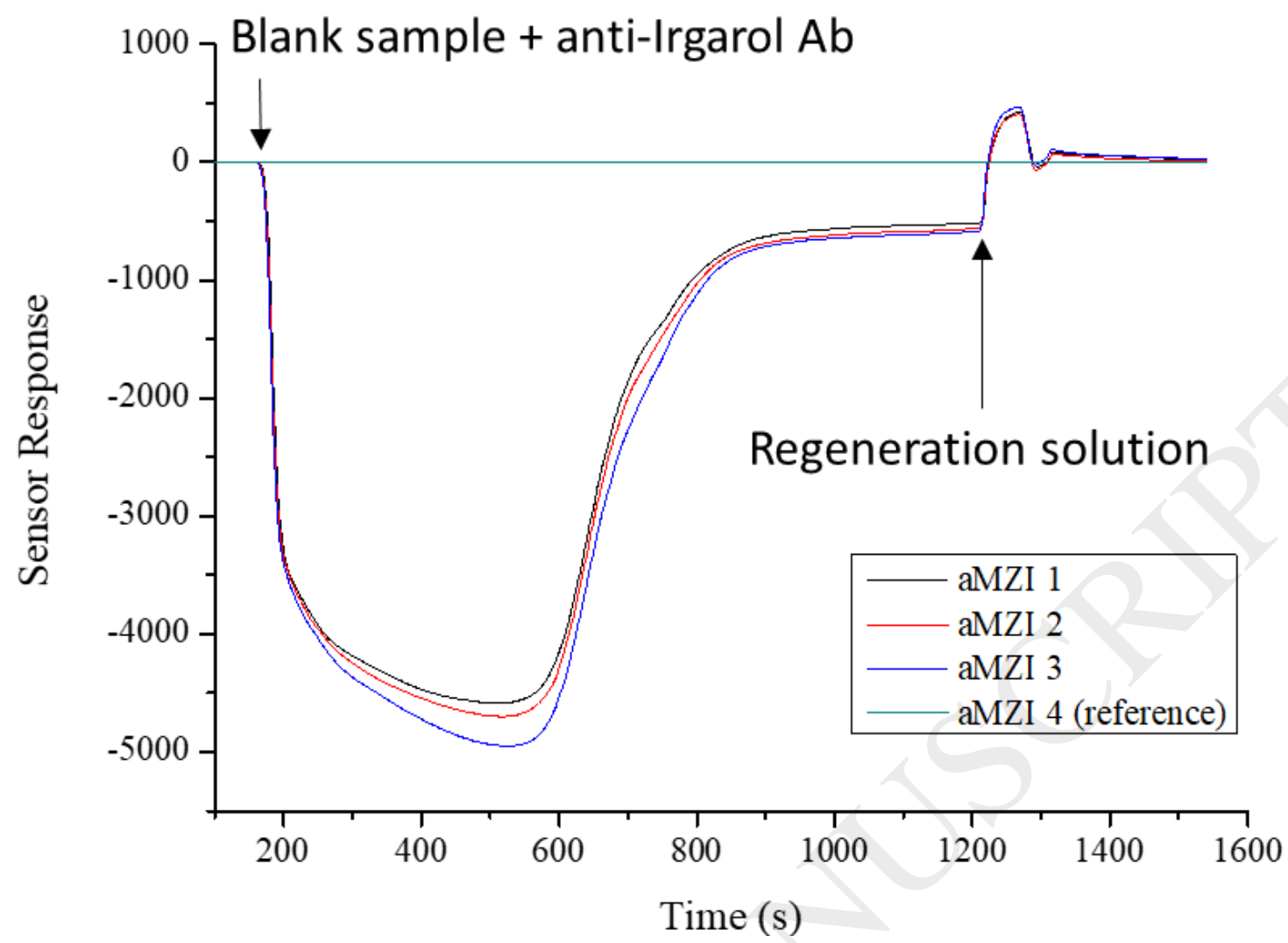

- Figure 13. Design of a Rotatory Valve fluidic glass chip. In light blue the fluidic channels, in turquoise the connections of the channels to the outside (opening at frontside of chip) and in orange the connection to the channels in the rotor (opening at backside of chip).

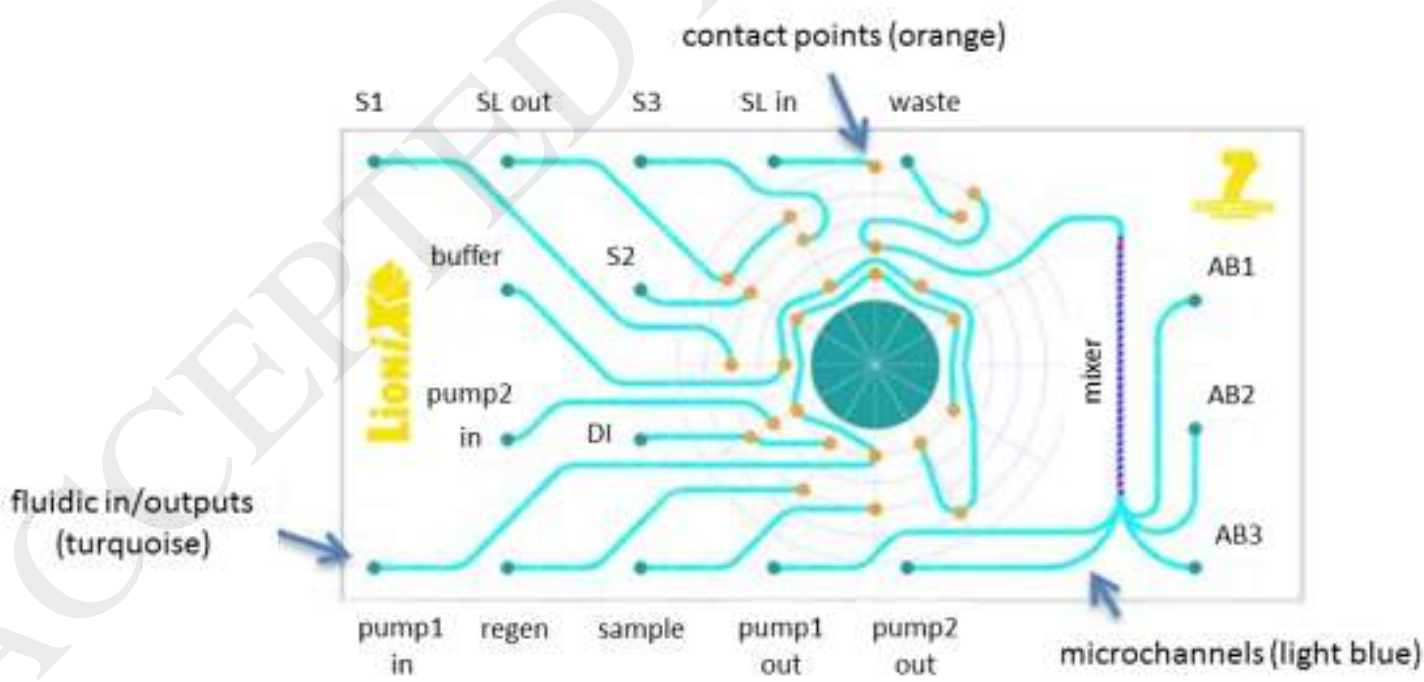

- Figure 14. Cross section of the Rotatory Valve showing the Teflon rotor pressed against the chip and providing a connection between two powderblasted holes in the glass chip. 


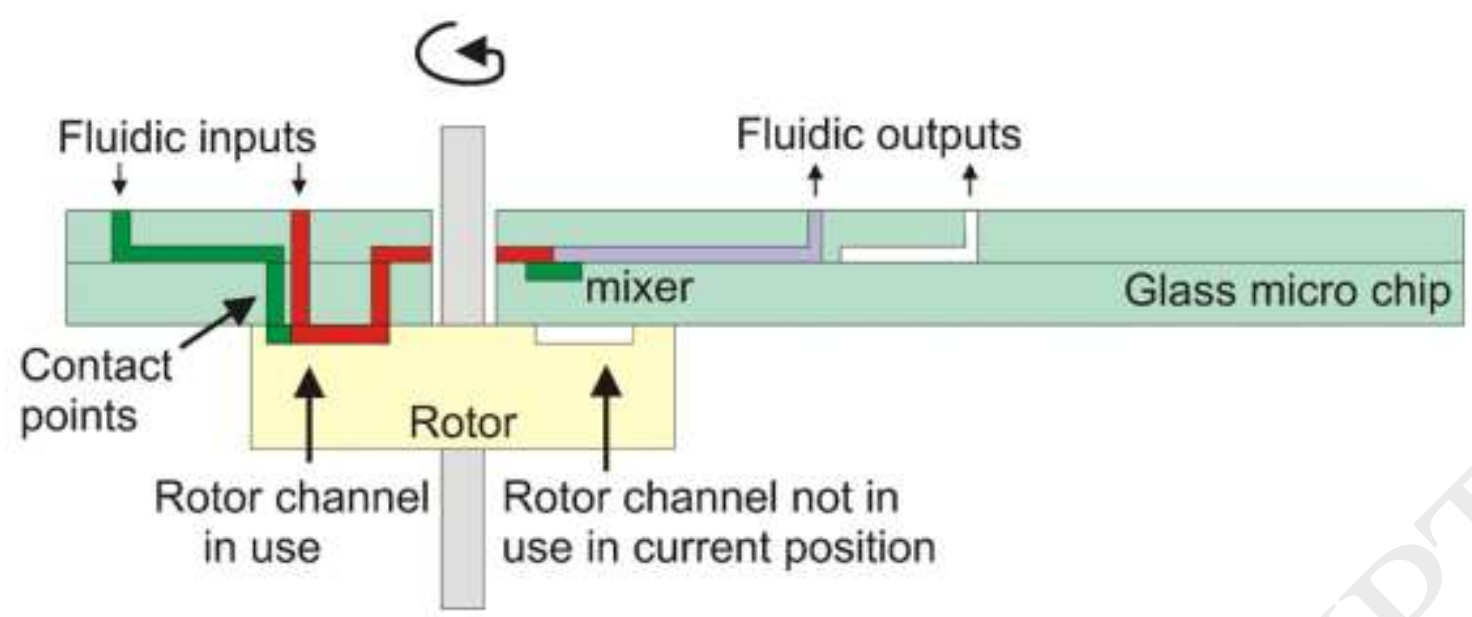

- Figure 15. An overview of the complete nano-immunosensor module. Left: Assembly of the RV. The stepper motor connected by gearings to the Teflon rotor next to the optical switch and "zero" position lever. The glass microchip is clamped in the Polyether ether ketone PEEK holder and not visible. Right: Complete module with the power supply, fluidics and communication lines of the buoy.

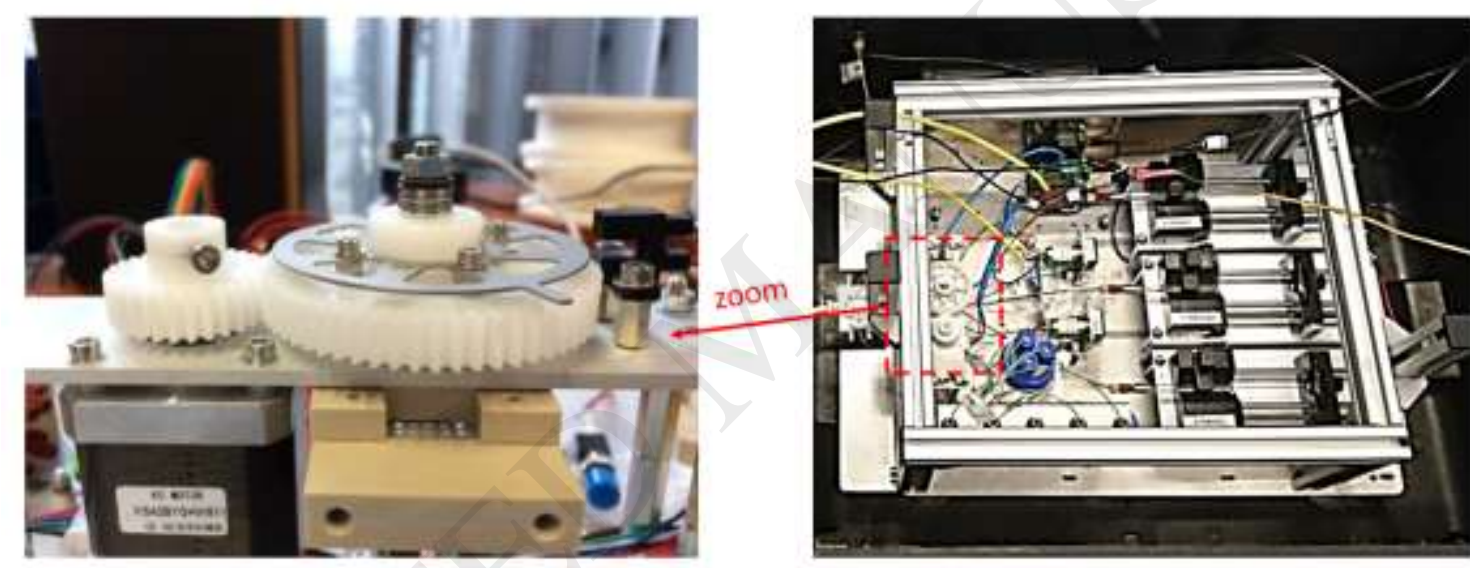

- Figure 16. Complete measurement cycle for a generic serum, from the mixture sample to the regeneration, by using the RV in automated mode. 


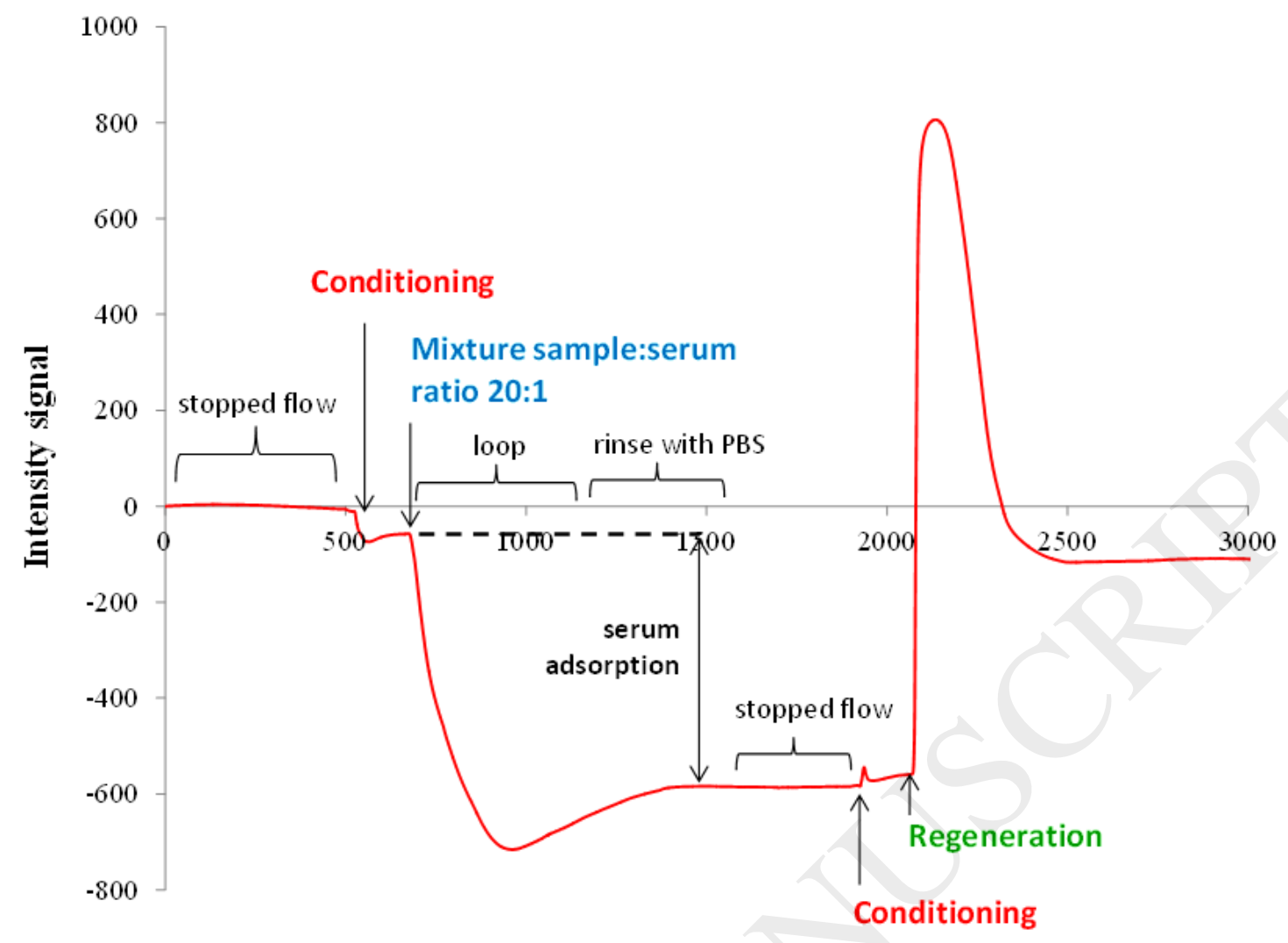

Time (s) 